\title{
The Adult Orthodontic Patient: More Options than Ever Before!
}

\section{Nour Eldin Tarraf*}

Department of Orthodontics, University of Sydney, Australia

\begin{abstract}
More and more adults are now presenting for orthodontic treatment. They don't only present with malocclusions of varying severity but also additional restorative and periodontal needs requiring well coordinated interdisciplinary care. In addition, today's adult patients also present with a demand for more aesthetic and less visible orthodontic appliances. This paper discusses the important considerations in managing adult orthodontic patients. It also highlights recent technological advances that enable us to better plan the management of adult patients as well as the available aesthetic treatment options and their strengths and weaknesses.
\end{abstract}

Keywords: Orthognathic surgery; Relapse patients; Bisphosphonates; Orthodontics

\section{Introduction}

Adult patients are composing an ever increasing demographic in orthodontic practice. According to the survey conducted by the Journal of Clinical Orthodontics in 2013 they composed $23 \%$ on average of orthodontic patients [1] as opposed to $10 \% 15$ years earlier. It is hard to determine exactly why more adults are seeking orthodontic treatment however some of that increase may be attributed to companies like Invisalign $^{\circledR}$ (Align Technology Inc, Santa Clara, Calif) marketing invisible orthodontic appliances directly to the public. This may have made more adults aware that they can in fact have orthodontic treatment and that it is not something that is limited to children and adolescents. It also made it more acceptable by providing a relatively invisible solution.

\section{Who are the Adult Patients?}

According to Proffit [2] there are two main groups of adults seeking orthodontic treatment. The first group is after some sort of adjunct orthodontic treatment to facilitate other dental work such as pre-prosthetic orthodontics, implant space preparations etc. These patients are usually middle aged adults in their 40s and 50s and most likely referred by their dental practitioner. The second group is adults that are after a comprehensive orthodontic treatment. They are usually younger adults who have always wanted orthodontic treatment but for some reason did not get it as teenagers and now they can afford it. However it is the author's opinion that a third group should now be considered namely the "relapse patients". A very large proportion of children and adolescents received orthodontic treatment in the 1970s, 80 s and 90s and in many of those the alignment, especially that of the lower anterior teeth [3], will have relapsed and they are now seeking a second, often simpler, orthodontic treatment to regain the alignment of the anterior teeth (Figure 1).

\section{Considerations in adult treatment}

When considering adult orthodontic treatment several factors must be taken into account. Firstly there is no more or very minimal growth [4] and the minimal amounts of growth that do occur would have no bearing on the outcome of orthodontic treatment. This makes the management of skeletal discrepancies more difficult and may require the inclusion of orthognathic surgery as an adjunct to orthodontic treatment. Secondly adults will usually present with a more extensive medical and dental history. In many cases adults are taking multiple medications some of which may have an influence on tooth movement. It is well documented that the chronic use of nonsteroidalanti-inflammatory drugs can slow down and sometimes inhibits orthodontic tooth movement [5]. In addition drugs that influence bone turnover, such as bisphosphonates used by many in the management of osteoporosis, can also inhibit tooth movement [6]. Therefore a thorough medical history is required and liaison with the managing medical practitioner may be necessary in some cases to see if an alternative drug can be used during orthodontic treatment.

It is well documented that orthodontic treatment in the presence of active periodontal disease can lead to rapid periodontal breakdown [7]. With adults there is an increased likelihood of active periodontal disease. The data from the recent national health survey from the United States shows that $47 \%$ of adults above the age of 30 will have some form of active periodontal disease [8]. Therefore thorough periodontal assessment should be conducted for every adult patient prior to any orthodontic treatment and active periodontal disease must be controlled first. However it is not contraindicated to treat adults with a history of periodontal disease provided the condition has been stabilized through periodontal therapy [9] and the patient is willing to attend regular maintenance throughout the treatment. Careful

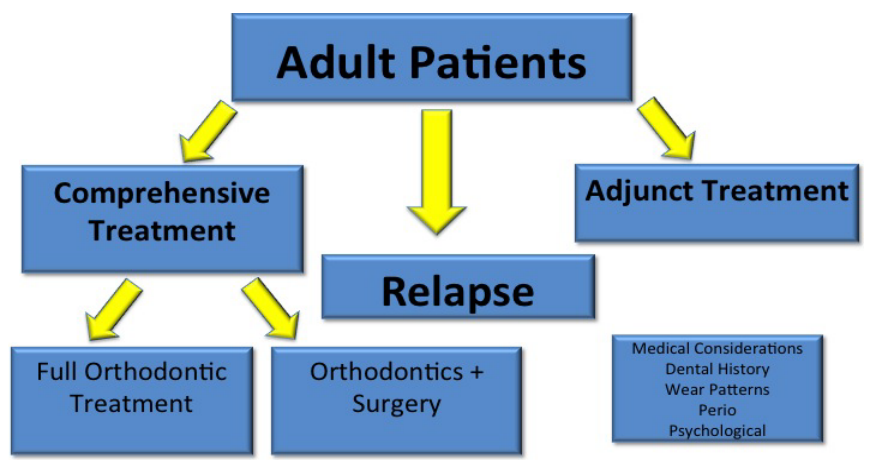

Figure 1: The three groups of adult patients as modified from Proffit et al. [2].

*Corresponding author: Nour Eldin Tarraf, PhD Candidate, Department of Orthodontics, University of Sydney, BDS (Hons), MDSc(Hons), MOrthRCSEd, MRACDS (Ortho), Private Practice, Chatswood, NSW, Australia, Tel: 029411 3836; E-mail: noureldintarraf@hotmail.com

Received October 28, 2014; Accepted January 11, 2015; Published January 16 2015

Citation: Tarraf NE (2015) The Adult Orthodontic Patient: More Options than Ever Before!. Dentistry 5: 278. doi:10.4172/2161-1122.1000278

Copyright: (๑) 2015 Tarraf NE. This is an open-access article distributed under the terms of the Creative Commons Attribution License, which permits unrestricted use, distribution, and reproduction in any medium, provided the original author and source are credited. 
adjustment of the orthodontic forces will be required in cases where there has been bone loss due to the apical migration of the centre of resistance of the tooth [10], which amplifies the moments generated by any forces applied to the crowns of the teeth [2].

\section{New technologies in adult treatment}

In recent years there have been several new advances, which have made a significant impact on adult orthodontic treatment. The introduction of digital three dimensional treatment planning tools, application of skeletal anchorage devices and more importantly the advances in invisible treatment techniques such as aligner treatments and lingual orthodontics have made adult orthodontic treatment much more streamlined and accessible.

As mentioned above [2] most adults will have some pre-existing restorations in addition to various wear facets that resulted from functioning with a malocclusion for a long period of time. Others are actually referred for orthodontic treatment in order to facilitate restorative treatment. It is therefore important to communicate to them why restorative treatment is required post orthodontic treatment to insure an aesthetic and functional result. In addition it is important for the restoring dentists to communicate to the orthodontists the desired final tooth positions to facilitate the best restorative outcomes. A variety of new 3D planning tools can now aid clinicians in both interdisciplinary planning as well as in explaining treatment requirements to patients. Digital diagnostic setups are invaluable in predicting treatment outcomes and communicating to the patients why there will be a need to have restorative work after orthodontics for example in the presence of extensive wear (Figure 2). Not only does it allows the patient to understand the treatment but also enables them to budget appropriately for it. The digital set-ups also allow the orthodontist to evaluate the type of tooth movement required to achieve the desired results and thus makes them able to select appropriately between fixed lingual appliances and/or aligner treatments. It also makes it possible to assess whether additional temporary anchorage devices (TADs) will be needed (Figure 2).

Cope in 2007 [11] defined them as: "(TAD) or a temporary anchorage device is a device that is temporarily fixed to bone for the purpose of enhancing orthodontic anchorage by supporting the teeth of the reactive unit or by obviating the need for the reactive unit altogether and which is subsequently removed after use". The introduction of TADs [11] has made it possible to provide treatment for adults with no or minimal posterior anchorage with reliable outcomes (Figure 3). It has also allowed tooth movements which were not possible with conventional appliances such as predictable molar intrusion in cases where molars have over erupted into long standing extraction spaces [12].

But it is perhaps the introduction of invisible treatment options that have been the most significant factor in revolutionizing adult treatment. Even though it is a lot more acceptable for adults nowadays to be wearing "braces" most adult patients - if given the choice - will prefer an invisible treatment option. Today's orthodontists have at the disposal two main appliance systems that will be considered invisible. Lingual fixed appliances and aligner treatments.

Lingual orthodontic appliances have been around since the late 1970s [13] but their popularity decreased somewhat in the 1990s. There were many reasons for that but technical difficulty and patient discomfort were some of the more commonly cited reasons. However the technique remained popular in Japan and Europe and there has been a rapid worldwide resurgence in the use of lingual appliances in the last decade [14]. This is mainly due to the introduction of a new generation of customized lingual appliances [15,16], which have overcome many of the limitations of previous generations by dramatically improving patient comfort and providing precision, predictability and versatility equivalent to that of labial appliances. For example in the Incognito ${ }^{\mathbb{B}}$ system (3M-Unitek, Monrovia, Calif) laboratory technicians fabricate a digital setup model according to the orthodontist's prescription (Figure 4). Brackets and wires are CAD/CAM customized on a digital model of the patient's final setup prior to the beginning of treatment.

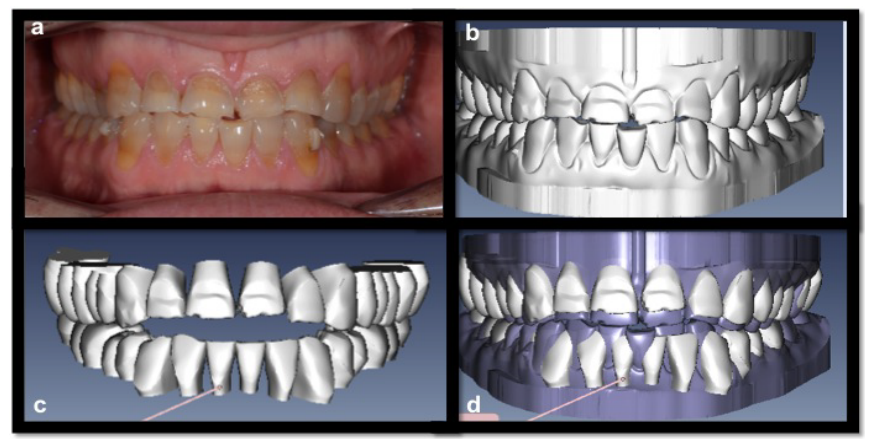

Figure 2: a) severely worn anterior teeth due to an edge to edge occlusion, b) digital malocclusion model, c) digital setup of tooth position required for restoration of lost tooth structure, d) digital model and setup superimposed to allow visualization of tooth movement required Images using TMP software (3M-Unitek, Monrovia, Calif).

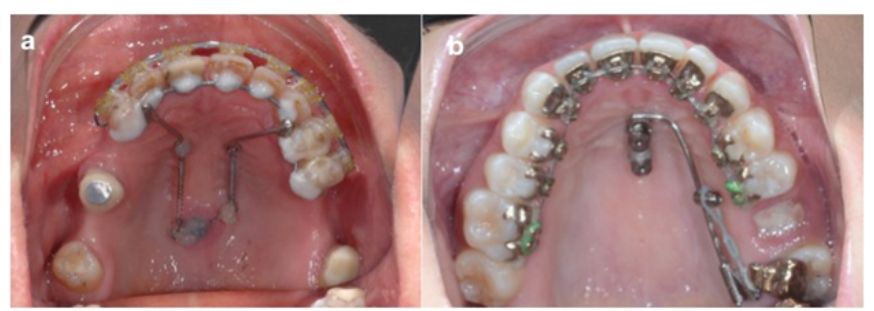

Figure 3: a) Palatal TADs being used to control the anterior segment in a patient lacking sufficient posterior teeth, b) palatal TADs being used as anchorage to move a second molar mesially to substitute an extracted first molar.

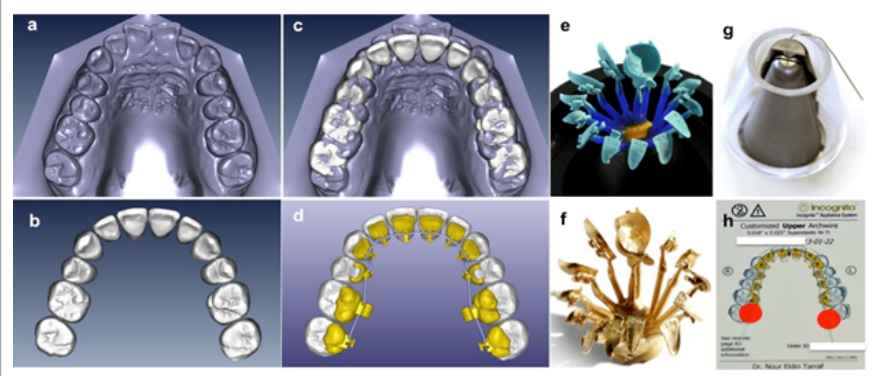

Figure 4: The steps in manufacturing of the customized lingual appliance Incognito ${ }^{8}$ (3M-Unitek, Monrovia, Calif) a) digital malocclusion model, b) set-up model based on orthodontist's prescription, c) set-up model and malocclusion model superimposed allows visualization of the required tooth movement. d) Virtual brackets designed with customized virtual wire as close as possible to the tooth surface to minimise appliance profile. e) Brackets are printed in was, $\mathrm{f}$ ) brackets casted in gold, $\mathrm{g}$ ) wire-bending robot manufacturing the custom wires, h) custom wire individual to the patient. (e,f and $g$ with permission from 3M-Unitek, Monrovia, Calif) 
These models are used as a template to design virtual brackets and wires. The virtual brackets are then printed through a high-end 3D printer in wax and then cast in a hard gold alloy. The arch wires are then custom-formed by a wire-bending robot. By providing a casted appliance that is made to mimic the individual anatomy of the patient's lingual surfaces the profile of the appliances is greatly reduced and the robotically bent wires insure accurate expression of the prescription (Figure 5). This has made the appliance much more comfortable as well as very precise [17] as shown by recent studies. In addition the Lingual appliances can now be used in combination with TADs, functional appliances and orthognathic surgery making them as versatile as labial fixed appliances [18-20]. In addition when compared to labial fixed appliances the Incognito ${ }^{\circledR}$ appliance (3M-Unitek, Monrovia, Calif) has been shown to cause less white spot lesions and enamel decalcification [21].

Aligner treatment is the more widely used option for adult treatment [22] with Invisalign ${ }^{\circledR}$ being the leading system in that field. Invisalign ${ }^{\circledR}$ introduced by Align Technology in 1997 was initially aimed at adult patients. The system allows for digital laboratory set-ups to be prepared by laboratory technicians and then the orthodontists can review the set-up through the ClinCheck ${ }^{\circledR}$ software and make any necessary changes. Then a series of plastic aligners is manufactured to sequentially move the teeth to the desired position throughout the course of the treatment.

Both types of appliances have their merits and demerits. They are not mutually exclusive and in order to successfully manage an adult focused orthodontic practice the practitioner needs to be competent in offering both. For example the Invisalign ${ }^{\circledR}$ appliance is a removable appliance and thus results can be highly dependent on patient compliance. The removable nature of the appliance also makes certain tooth movement less predictable [23] and although successful extraction treatment with aligners has been reported in the literature [24], control of root position, especially when large extraction spaces are being closed, remains a weakness of the appliance. Fixed lingual appliances will certainly be more suitable for precise control in those cases (Figure 6) [25]. Another limitation is the inability to restore teeth while in active treatment as changes in tooth anatomy will make subsequent aligners not fit. In those cases the lingual appliances are favored as anterior restoration can be started while the posterior occlusion is being detailed making it possible for the patients to enjoy a more aesthetic appearance early on in treatment. In addition the Invisalign $^{\circledR}$ aligners still cover the labial surface of the teeth and thus it can be considered a semi invisible appliance especially with the addition of attachments to improve tooth control. This may not meet the aesthetic demands of some adult patients. Lingual appliances on the other hand can offer a truly hidden appliance (Figure 7). However one of the major strengths of the Invisalign ${ }^{\circledR}$ appliance is the ability to isolate certain teeth in treatment and to reduce the rate of tooth movement of those teeth through the ClinCheck ${ }^{\circledR}$ software. This is of particular advantage when dealing with periodontally compromised teeth as the rate of tooth movement can be reduced on certain affected teeth over others. The removable nature of the appliance also allows for better hygiene management during active treatment [26]. Furthermore the oral discomfort associated with the Invisalign ${ }^{\circledR}$ appliance is the least reported when compared to fixed appliance treatments [27] and this can be a major advantage for many patients especially those with less extensive malocclusions. It is also important to consider combining the various systems to tailor the treatment to malocclusions of various severities. There is no reason why one arch could not be treated with lingual appliances while the other arch treated with aligners depending on the complexity of the tooth movement required.

\section{Conclusion}

In conclusion it can be said that with increasing numbers of adults demanding orthodontic treatment orthodontists are now better equipped to offer an aesthetic treatment option for almost any malocclusion. The practitioner can now select and combine different technologies to customize a treatment solution that can deliver the tooth movement required while still fitting the patient's aesthetic requirements.

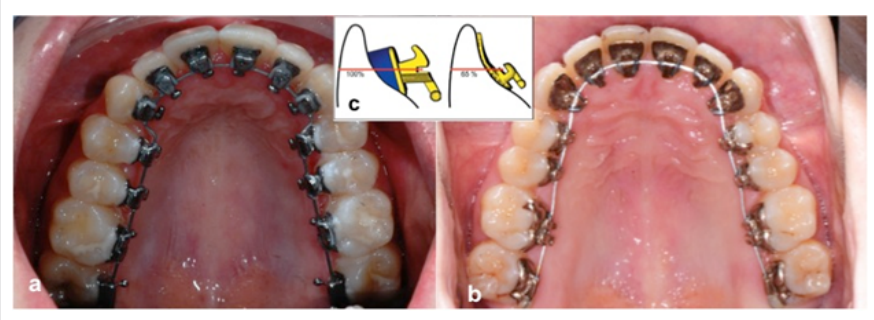

Figure 5: Comparison between a conventional lingual bracket with composite pads (Figure $5 \mathrm{a}$ ) and casted custom made (Figure $5 \mathrm{~b}$ ) Incognito $₫$ appliance (3M-Unitek, Monrovia, Calif). c) The custom appliance is around $35 \%$ smaller in profile thus less obtrusive to the tongue.

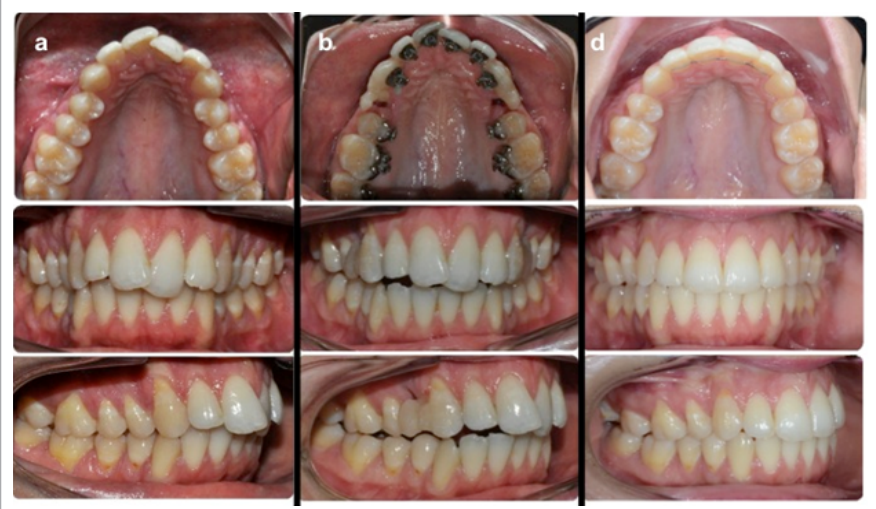

Figure 6: Extraction treatment with upper premolar extraction to camouflage a severe Class II div 1 malocclusion. Composite pontics are built to cover extraction site during space closure. This is a case demonstrated the superior control lingual fixed appliances can offer in complex cases. This level of control is difficult to achieve with aligner treatment.
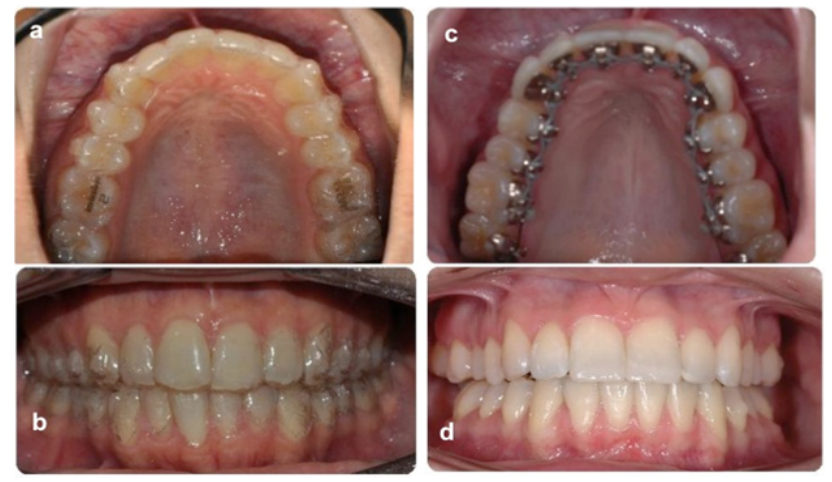

Figure 7: Comparison of aligner appearance versus lingual appliances. a and b) Aligners are semi invisible, c and d) lingual fixed appliances completely invisible. 


\section{References}

1. Keim RG, Gottlieb EL, Nelson AH, Vogels DS (2013) Orthodontic Practice Study. Part 1: trends. Journal of Clinical Orthodontics; JCO 47: 661-680.

2. Proffit W, Jr HF, Sarver D (1999) Contemporary Orthodontics (4thedtn). Elsevier, Philadelphia, USA.

3. Little RM (1999) Stability and relapse of mandibular anterior alignment: University of Washington studies. Semin Orthod 5: 191-204.

4. Behrents RG (1985) The biological basis for understanding craniofacial growth during adulthood. Prog Clin Biol Res 187: 307-319.

5. Bartzela T, Turp JC, Motschall E, Maltha JC (2009) Medication effects on the rate of orthodontic tooth movement: a systematic literature review. American Journal of Orthodontics and Dentofacial Orthopedics 135: 16-26.

6. Zahrowski JJ (2009) Optimizing orthodontic treatment in patients taking bisphosphonates for osteoporosis. American Journal of Orthodontics and Dentofacial Orthopedics 135: 361-374.

7. Wennstrom JL, Stokland BL, Nyman S, Thilander B (1993) Periodontal tissue response to orthodontic movement of teeth with infrabony pockets. American Journal of Orthodontics and Dentofacial Orthopedics 103: 313-319.

8. Eke PI, Dye BA, Wei L, Thornton-Evans GO, Genco RJ (2012) Cdc Periodontal Disease Surveillance workgroup: James Beck GDRP. Prevalence of periodontitis in adults in the United States: 2009 and 2010. J Dent Res 91: 914-920.

9. Polson A, Caton J, Polson AP, Nyman S, Novak J et al. (1984) Periodontal response after tooth movement into intrabony defects. J Periodontol 55: 197202.

10. Melsen B, Agerbaek N, Markenstam G (1989) Intrusion of incisors in adult patients with marginal bone loss. American Journal of Orthodontics and Dentofacial Orthopedics 96: 232-241.

11. Cope JB (2007) Ortho TADs: The Clinical Guide and Atlas. Under Dog Media, LP, Dallas, Texas.

12. Yao C, Lee J, Chen H, Chang Z, Chang H, et al. (2005) Maxillary Molar Intrusion with Fixed Appliances and Mini-implant Anchorage Studied in Three Dimensions. Angle Orthod 75: 754-760.

13. Alexander CM, Alexander RG, Gorman JC (1982) Lingual orthodontics. A status report. J Clin Orthod 16: 255-262.
14. Keim RG (2012) The resurgence of lingual orthodontics. J Clin Orthod 46:197198.

15. Ludwig B, Alexander JC, Cacciafesta V (2012) Lingual orthodontics. Part 1. J Clin Orthod 46: 203-217.

16. Takemoto K, Scuzzo G (2001) The straight-wire concept in lingual orthodontics. J Clin Orthod 35: 46-52.

17. Grauer D, Proffit WR (2011) Accuracy in tooth positioning with a fully customized lingual orthodontic appliance. Am J Orthod Dentofacial Orthop 140: 433-443.

18. Park HS (2006) A miniscrew-assisted transpalatal arch for use in lingual orthodontics. J Clin Orthod 40: 12-16.

19. Pauls HJ (2008) Lingual orthodontics with orthognathic surgery in a severe class II division 2 case. J Orofac Orthop 69: 135-145.

20. Wiechmann D, Schwestka-Polly R, Hohoff A (2008) Herbst appliance in lingual orthodontics. Am J Orthod Dentofacial Orthop 134: 439-446.

21. Vander Veen MH, Attin R, Schwestka-Polly R, Wiechmann D (2010) Caries outcomes after orthodontic treatment with fixed appliances: do lingual brackets make a difference? European Journal of Oral Sciences 118: 298-303.

22. Vicens J, Russo A (2010) Comparative use of Invisalign by orthodontists and general practitioners. Angle Orthodontist 80: 425-434.

23. Kravitz ND, Kusnoto B, BeGole E, Obrez A, Agran B (2009) How well does Invisalign work? A prospective clinical study evaluating the efficacy of tooth movement with Invisalign. American Journal of Orthodontics and Dentofacial Orthopedics 135: 27-35.

24. Honn M, Goz G (2006) A premolar extraction case using the Invisalign system. Journal of Orofacial Orthopedics 67: 385-394.

25. Uribe F, Cutrera A, Nanda R (2011) A segmented appliance for space closure followed by Invisalign and fixed appliances. Orthodontics: The Art and Practice of Dentofacial Enhancement 12: 386-395.

26. Miethke RR, Brauner K (2007) A Comparison of the periodontal health of patients during treatment with the Invisalign system and with fixed lingual appliances. Journal of Orofacial Orthopedics 68: 223-231.

27. Miller KB, McGorray SP, Womack R (2007) A comparison of treatment impacts between Invisalign aligner and fixed appliance therapy during the first week of treatment. American Journal of Orthodontics and Dentofacial Orthopedics 131: 302.e1-302e9. 